\title{
A Acupuntura no Ocidente
}

\author{
Guido Palmeira *
}

$O$ reconhecimento da eficácia da acupuntura nāo depende da demonstração empírica de seus resultados. Problemas metodológicos e conceituais dificultam o estabelecimento de seu valor terapêutico, com base na ciência ocidental moderna.

Por outro lado, o crescimento da demanda e da oferta de terapias alternativas (entre elas a acupuntura) implica uma certa legitimação, que depende mais do reconhecimento da utilidade dessas práticas, do que da demonstração de sua cientificidade. A crise da "medicina cientifica" e de seu paradigma mecanicista pode ser um dos fatores responsáveis pela maior aceitação da acupıntura no Ocidente. Se isto é verdade, os estudos cientificos sobre acupuntura serão de pouca utilidade, enquanto persistirem em negar a possibilidade de uma medicina que tem a sua lógica própria, diferente daquela da ciência ocidental. Talvez a maior colaboração que o Oriente possa trazer à medicina ocidental nāo esteja na sua técnica, mas no seu saber. No entanto, é apenas através da compreensão da cultura e da civilização chinesas, da aceitaçāo de que Yin e Yang se organizam em um sistema coerente, que o saber tradicional pode ser realmente apreendido.

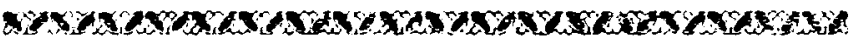

\section{INTRODUÇÃO}

Temos assistido a um crescente interesse pelas chamadas "práticas al temativas de saúde". Sob esta designação genérica, destacam-se, pela freqüência com que são mencionados, especificamente, o uso de plantas medicinais, a homeopatia e a acupuntura.

No Brasil, a acupuntura já vinha sendo incorporada como alternativa terapêutica, em geral associada a procedimentos da medicina científica ocidental, em vários hospitais universitários, desde o início dos anos 80. A homeopatia foi reconhecida como especialidade médica pelo Conselho Federal de Medicina em 1980, passando a ser oferecida como opção terapêutica, em algumas unidades da Previdencia Social, desde 1986.

Cademos de Saúde Pública, RJ, 6 (2) : 117-128, abr/jun, 1990 
Durante o último Congresso Brasileiro de Saúde Coletiva, o assunto mereceu destaque através de "Comunicações Coordenadas". Sob o título geral de "Proposições altemativas de assistência à saúde", discutiram-se a história e a "fundamentação científica" da homeopatia e da acupuntura, as experiências de implantação dessas práticas na rede pública, em vários Estados da Federação, e os problemas existentes para estender as 'práticas altemativas' ao SUS.

Vale observar que a oferta de "terapias altemativas" pelo serviço público foi precedida da multiplicação de consultórios privados de homeopatia e acupuntura, o que supōe a existência de uma demanda específica a este tipo de terapia, por parte de uma parcela da população que pode pagar por serviços de saúde privados. Por outro lado, a aceitação dessas práticas, pelos usuários dos serviços públicos, tem mostrado que esta demanda não é exclusiva daquela parcela da população.

A oferta de tratamentos por ervas, homeopatia ou acupuntura por serviços públicos de saúde supõe o reconhecimento oficial de sua utilidade. Esse reconhecimento vem sendo buscado e, em certa medida, tem sido conquistado, através de diversos argumentos e estratégias. Pretende-se, neste artigo, discutir, no caso específico da acupuntura, algumas questões decorrentes dos caminhos pelos quais se tem buscado sua legitimação no ocidente. Cabe observar que, uma vez que as estratégias para a legitimaçāo são as mesmas, $\tan$ to para a acupuntura quanto para a homeopatia e para o uso de ervas medicinais, considerações semelhantes também poderiam ser feitas em relação a estas práticas.

\section{O APELO À DEMONSTRAÇÃO EMPÍRICA DO SUCESSO DO MÉTODO}

O relato de curas, muitas vezes espetaculares, com o uso da acupuntura, tem sido recurso freqüente. O sucesso da anestesia com acupuntura, em diferentes cirurgias, tem produzido um grande impacto no ocidente desde a década de 70; os casos observados por Bland foram, nas suas próprias palavras, "suficientes para provar o valor da acupuntura como tratamento e como anestésico." (Bland, 1979).

Verifica-se, no entanto, que a demonstração empírica dos resultados obtidos com a acupuntura, por si só, tem se mostrado insuficiente para o reconhecimento da sua eficácia terapêtutica, pois tais resultados são interpretados pelos céticos como embuste ou, na melhor das hipóteses, como conseyüência de pura sugestão; segundo estes, as agulhas agiriam, no máximo, como placebo.

Cademos de Saúde Pública, RJ, 6 (2) : 117-128, abr/jun, 1990 
A preocupação de mostrar que os resultados obtidos com a acupuntura não se devem a sugestão está presente no discurso de Huan Xiang Ming (vice-diretor do Instituto de Pesquisa Médica Chinesa, em Xangai), em um seminário patrocinado pela OMS na China, em 1979 (ver: A Saúde do Mundo 12/79): "o êxito da anestesia por acupuntura e a cura da disenteria bacilar pela acupuntura abalaram a opinião de que o efeito desse procedimento não passa de uma ilusâo psicológica" (Huan Xiang Ming, 1979), ou, nas palavras de Bland: “... mas se a funçâo anestésica da acupuntura é 'puramente mental' (aspas no original), como explicar que as agulhas parecem ser igualmente eficientes na veterinária?" (Bland, 1979).

$\mathrm{Se}$, por um lado, o ceticismo continua presente no ocidente, de outro, muitos autores ocidentais já compartilham da opiniāo de que "... o número de casos estudados fornece alguma indicação da presença de um fenômeno que requer investigaçâo adicional". (Patel, 1987).

\section{A BUSCA DE UMA 'EXPLICAÇÃO CIENTÍFICA' PARA OS EFEITOS E MECANISMOS DE AÇÃO DA ACUPUNTURA}

A tentativa de demonstrar a cientificidade da acupuntura é tarefa a que vêm se dedicando inúmeros acupuntores, desde o início do século. As páginas preliminares de "L'Acupuncture Chinoise", publicada na França por Soulié de Morant, em 1939, e que marcou o renascimento do interesse pela acupuntura no ocidente, já mostram certa preocupação neste sentido. Os trabalhos de Noboyet, demonstrando a diferença da resistência elétrica da pele nos pontos de acupuntura, "que permitiu a deteç̧ão dos pontos por multivol. timetros"' (Cintract, 1982), foram publicados, inicialmente, em 1955.

Neste período, a medicina científica alcançava grande prestígio. A descoberta dos antibióticos, das vacinas e do DDT levavam à crença de que, com o desenvol vimento da tecnologia, seriam erradicados, um a um, os agentes etiológicos específicos de cada uma das doenças conhecidas. O desenvolvimento da acupuntura e sua credibilidade dependiam, neste contexto, de que se pudesse demonstrar alguma cientificidade no método das agulhas.

Entre 1912 e 1949 , mesmo na China, verificaram-se tentativas de eliminar a prática da medicina tradicional, sob a alegação de que não tinha bases científicas. Segundo Cai Jing Feng, “... antes da fundação da Nova China, em 1949, o conflito entre a medicina tradicional chinesa e a medicina ocidental foi, basicamente, a luta do sistema tradicional em con-

Cadernos de Saúde Pública, RJ, 6 (2) : 117-128, abr/jun, 1990 
(*) Meridianos são canais virtuais que percorrem todo o corpo, e pelos quais circula a energia vital $\mathrm{Ch}^{\prime} \mathrm{f}$. tinuar existindo, contra a idéia reacionária e subjetiva de que o sistema tradicional era retrógrado e náo-cientifico", (Cai Jing Feng, 1988).

Após a revolução socialista de 1949 , a política oficial do govemo chinês passa a ser a da integração entre os dois sistemas. Os médicos chineses, de estilo ocidental', são encorajados a aprender a medicina tradicional e, ao mesmo tempo, incentivados a estudá-la com base em métodos científicos modernos. Um dos artigos da constituição chinesa estipulava que: " $A$ nação, no desenvolvimento de cuidados de saúde $e$ de programas de higiene, desenvolveria a medicina moderna e a tradicional". (citado por Cai Jing Feng, 1988).

Em 1979, Huan Xiang Ming declarava (no seminário já referido) que "A pesquisa científica voltou (com Hua Kuo Feng) a ser chamada a desempenhar uma atividade fundamental em nossa construção socialista e para nossas quatro modernizaçōes; o campo da pesquisa acadêmica volta a recender a doçura da primavera" (Huan Xian Ming, 1979), e Bland falava dos estudos desenvolvidos na China "envolvendo o emprego de aparelhos eletrônicos altamente complexos... (que) estão se aprofundando nos mistérios das endorfinas." (Bland, 1979).

Aos poucos, a resistência inicial ao emprego da acupuntura, no ocidente, vai sendo substituída pela opiniāo de que é vantajosa a integraçāo entre os dois sistemas, o progresso da integração do conhecimento tradicional com o método científico" é visto por alguns representantes da academia ocidental como "uma grande promessa". (Kao, 1979).

Se, durante a primeira metade do século, os estudos 'científicos' procuravam, sobretudo, a confirmação da existência dos meridianos $\left(^{*}\right)$ e pontos descritos pela tradição, através da sua demonstração anatômica, a grande maioria dos estudos mais recentes é de ensaios clínicos que procuram medir a eficácia da acupuntura no tratamento de patologias específicas, ou de investigaçōes que buscam elucidar os mecanismos de ação das agulhas, principalmente pela identificação de substâncias neurotransmissoras envolvidas nos fenômenos de analgesia e anestesia com acupuntura. Os artigos relacionados no Index Medicus dos primeiros cinco meses de 1989 , a maioria publicada em revistas chinesas ou de países da Europa oriental, mostram claramente esta tendência.

Os estudos publicados no ocidente, na maioria dos casos ensaios clínicos onde se busca avaliar a eficácia da acupuntura no tratamento de dores crônicas de diferentes etiologias e localizações anatômicas, em geral, têm apresentado importantes deficiências metodológicas. (Lewith, 1984 e Patel, 1987). 
Muitos estudos foram realizados com pequeno número de pacientes, prejudicando a aplicação de testes de significância estatística. Em muitos casos, os critérios para a seleção inicial dos pacientes a serem incluídos no estudo são imprecisos ou maldefinidos; em outros, os critérios para a definição do que deve ser considerado sucesso ou falha do tratamento não foram bem estabelecidos desde o início do estudo.

Um dos freqüentes problemas metodológicos está na dificuldade de se obterem estudos cegos. Se o grupo-controle for submetido a procedimentos terapêuticos, claramente distintos da acupuntura (tratamento médico ocidental, injeção de anestésicos e/ou esteróides etc), tanto pacientes como terapeutas têm, necessariamente, conhecimento do grupo (experimental ou controle) a que cada membro do estudo pertence. Ao contrário do que ocorre com a comparação entre duas drogas, cujas apresentações podem ser indistintas, quando se utiliza a própria acupuntura como placebo (agulhas inseridas fora dos pontos de acupuntura) é impossível impedir que o terapeuta, um acupuntor que conhece a localizaçâo precisa dos pontos, identifique os pacientes que pertencem ao grupo experimental, que recebem o tratamento adequado, e aqueles que compõem o grupo-controle, tratados com 'acupuntura placebo'.

Depois de chamar a atenção para o fato de que a inserção aleatória das agulhas não tem apenas um efeito placebo, Lewith afirma que "...é dificil definir um placebo válido que pode ser usado, consistentemente, sem o conhecimento dos terapeutas"; e acrescenta que, dadas as diferenças individuais dos pacientes no que tange à resposta ao tratamento com acupuntura, os ensaios cruzados são "virtualmente impossiveis", dificultando que os estudos possam ser definidos em bases comparáveis.

Enquanto a acupuntura tradicional baseia o tratamento no entendimento tradicional da doença, seleciona os pontos a serem picados em função das necessidades individuais dos pacientes e, no curso do tratamento, modifica os pontos utilizados, segundo a variação das necessidades dos doentes, na maioria dos estudos examinados por Patel, os pontos são selecionados com base no diagnóstico da medicina científica. Identifica-se um conjunto de pontos definidos a priori como indicados para a patologia em questāo, que serão utilizados à maneira de uma fórmula ou prescrição que nāo varia de um doente para outro, e que não é modificada durante o tratamento. Considera-se que tais diferenças podem prejudicar, significativamente, os resultados do método tradicional.

O mesmo autor chama a atenção para o fato de que a maioria dos ensaios tem como objetivo avaliar

Cadernos de Saúde Pública, RJ, 6 (2) : 117-128, abr/jun, 1990 
os efeitos da acupuntura sobre a dor, embora o principal objetivo da medicina tradicional seja a prevenção da doença e a manutenção da saúde, restando ao tratamento da doença um papel secundário e, ao alívio da dor, uma função apenas complementar; e sugere a utilização de indicadores que possam medir o "estado de saúde global' dos pacientes, a principal precupação da terapêutica tradicional.

Os dois autores, Lewith e Patel, consideram que os estudos desenvolvidos no ocidente são insuficientes para estabelecer, definitivamente, o valor terapêutico ou analgésico da acupuntura. Por outro lado, ambos concordam em que é necessário realizar estudos mais bem elaborados, seja para justificar a eficácia da acupuntura como tratamento, seja como uma resposta da comunidade científica ao crescimento da utilização da medicina alternativa.

Embora os estudos científicos não permitam conclusões definitivas, o reconhecimento de vantagens da acupuntura tem sido um argumento em favor de sua utilização no ocidente. A partir dos anos 60 , o prestígio da medicina científica começava a ser abalado, as campanhas de erradicação, iniciadas na década de 50, com raras exceçōes, haviam malogrado (a este respeito, ver Yekutiel, 1981); a idéia de causa única, nascida no início do século, da demonstração de agentes etiológicos específicos para cada uma das doenças infecciosas, vinha se mostrando inadequada para a compreensão das doenças crônico-degenerativas, que assumiam importância cada vez maior no quadro nosológico dos países industrializados.

A ciência médica reconsiderou a importância dos fatores ambientais e sócio-culturais na determinação das doenças. As patologias crônico-degenerativas passaram a ser descritas em termos de distúrbios celulares; para seu estudo, diagnóstico e tratamento, foi necessário aumentar a precisão tanto da medição de constantes vitais, como da identificação de metabólitos específicos, envolvidos na gênese de diferentes patologias. A tecnologia médica desenvolveu instrumentos de investigação diagnóstica cada vez mais sofisticados e caros. O objetivo de erradicação das doenças transferiu-se para o da extensão de cobertura de serviços de saúde; ganhava espaço a idéia de que a saúde é funçāo da assistência médica individual; e o acesso aos serviços de saúde passaram a ser reivindicados por um contingente cada vez maior da população.

Segundo Bland, um delegado da Europa ocidental, no mesmo seminário de 1979 (ver: A Saúde do Mundo 12/79), declarava que: "... a forma praticável de introduzir serviços gerais de saúde, em condições, de atender a todos no ano 2000", seria através de "médicos descalços", que em "... um curso de 18 
meses... aprenderiam as técnicas de acupuntura, o emprego de ervas medicinais e outras formas de tratamento... trabalhariam em áreas rurais, e constituiriam a linha de vanguarda contra a doença" (citado por Bland, 1979).

\section{A LEGITIMAÇĀO DAS MEDICINAS ALTERNA- TIVAS E A CRISE DA MEDICINA CIENTÍFICA}

Apesar das dificuldades em provar seu valor terapêutico com suficiente rigor científico, o crescimento da demanda e da oferta de terapias alternativas (inclusive pelo serviço público) implica uma certa legitimação não-acadêmica dessas práticas. Existem indícios de que, hoje, a legitimação das práticas alternativas não depende apenas do reconhecimento de sua cientificidade, mas também do reconhecimento de sua utilidade terapêutica; ao contrário do que era dado pensar durante a primeira metade do século, quando o reconhecimento da utilidade terapêutica de qualquer método estava intimamente relacionado ao reconhecimento de sua cientificidade.

Aparentemente, tal mudança está relacionada à crise da medicina moderna. Se, por um lado, como veremos, alguns autores identificam a crise da medicina científica com a crise de seu paradigma, por outro, as práticas alternativas estão orientadas por paradigmas suficientemente distintos daqueles da medicina científica, para que sejam identificadas exatamente como "alternativas" a esta última.

Queiroz fala de uma "crise profunda" da prática e do saber da ciência médica moderna, que “... se refere à crise de seu paradigma dominante... o positivismo", e cujo sintoma principal é "... produzir servicos extremamente caros e ineficazes", mostra que "... historicamente, o desenvolvimento da medicina (cientifica) implicou a perda de uma visão unificadora do paciente, e deste com seu meio ambiente fisico e social" e que este é "... um fenômeno recente e sem similar, quando confrontado com sistemas médicos não-ocidentais: Nesses sistemas médicos alternativos... o fator social existe como componente fundamental, ao contrário do que ocorre com o paradigma dominante da medicina ocidental moderna."(Queiroz, 1986)

Capra também reconhece, na "influência do paradigma cartesiano sobre o pensamento médico" a origem dos problemas da moderna medicina científica: .... ao reduzir a saúde a um funcionamento mecânico, (a medicina moderna) nâo pode mais ocupar-se com o fenêmeno da cura... a prática médica, baseada em tâo limitada abordagem (a cartesiana) não é muito eficaz na promoção e manutenção da boa saúde. De

Cadernos de Saúde Pública, RJ, 6 (2) : 117-128, abr/jun, 1990 
fato, essa prática, hoje em dia, causa freqüentemente mais sofrimento e doença, segundo alguns autores (cita Illich), do que a cura." (Capra, 1986).

Lembra que “... a crescente dependência da medicina em relaçāo à alta tecnologia suscitou um certo número de problemas que não são apenas de natureza médica ou técnica, mas envolvem questões sociais, econômicas e morais muito mais amplas". Fala do desenvolvimento da quimioterapia e do uso inadequado e abusivo de medicamentos, que "... tornaram-se um problema de saúde pública de alarmantes proporções"; da relação entre a complexificação tecnológica do ato médico e a proliferação de especialidades médicas, que “... reforçou a propensão dos médicos de tratar partes específicas do corpo, esquecendo-se de cuidar do paciente como um ser total"; e da transferência da prática médica “... do consultório do clínico geral para o hospital, onde se tornou progressivamente despersonalizada, quando não desumanizada."

Não deixa de referir-se à "impressionante desproporção entre o custo e a eficácia da medicina moderna", destacando que "As intervenções biomédicas, embora extremamente úteis em emergências individuais, têm muito pouco efeito sobre a saúde de populações inteiras"; nem às doenças crônico-degenerativas, tidas pela ciência médica "... como conseqüência inevitável do 'desgaste' geral, para o qual não existe cura" (Capra, 1986).

Considera ainda que, como conseqũência $\mathrm{da}$ ".... imagem pública do organismo humano... uma máquina propensa a constantes avarias se não for supervisionada por médicos e tratada com medicamentos", não são valorizadas “... a noção do poder de cura inerente ao organismo e a tendência para manter-se saudável, ... a confiança do individuo em seu próprio organismo, ... (ou) a relação entre saúde e hábitos de vida."

O mesmo autor, no capítulo em que trata do "Holismo e Saúde", acredita que "... podemos aprender com os modelos médicos existentes em outras culturas." Embora não seja seu objetivo apresentar a medicina chinesa como prática ideal, e apesar de advertir que "as comparações entre sistemas médicos de diferentes culturas devem ser feitas com todo o cuidado", reconhece que "... a noçâo chinesa do corpo como um sistema indivisivel de componentes intercalados está, obviamente, muito mais próxima da abordagem sistêmica do que o modelo cartesiano clássico." Ressalta que, para a medicina chinesa, "... a doença não é considerada um agente intruso, mas o resultado de um conjunto de causas que culminam em desarmonia $e$ desiquilíbrio... (e) será, em dados momentos, inevitável no processo vital... a saúde perfeita não é o objeti- 
vo essencial... o papel principal dos médicos chineses sempre foi o de evitar o desequilíbrio de seus pacientes." Observa que as diferentes técnicas terapêuticas da medicina chinesa visam "... estimular o organismo do paciente de tal modo que ele siga sua própria tendência natural para voltar a um estado de equilíbrio. Assim, um dos principais principios da medicina chinesa é sempre administrar uma terapia a mais branda possivel"' (Capra, 1986).

Se a crescente aceitação da acupuntura, no ocidente, está relacionada à crise da medicina científica; se, em conseqüência do alto custo da medicina tecnológica, é preciso referendar práticas não totalmente legitimadas pela ciência médica, com o argumento de que são úteis, mais simples e mais baratas $\left({ }^{*}\right)$; se a procura de terapias em princípio 'mais brandas' pode ser vista como resposta à agressividade da intervenção médica tecnologizada; se a procura por uma medicina, cujos princípios são considerados 'mais holísticos' (Capra), pode significar uma reação à segmentação do olhar da ciência médica (explicitado pela profusão de especialidades e subespecialidades), ou mesmo à desumanização da prática médica hospitalar; então a questão que se coloca não é mais a da investigação dos mecanismos de ação da acupuntura, mas a investigação de seus princípios, de sua lógica, de seus paradigmas.

\section{A INTEGRAÇÃO DOS DOIS SISTEMAS NO OCI- DENTE}

A acupuntura é apenas uma das técnicas terapêuticas que compõem um conjunto de saberes e procedimentos culturalmente constituídos, e dos quais não pode ser dissociada. Além das agulhas, a medicina tradicional utiliza ervas, massagens, exercícios físicos, dietas alimentares, e prescreve normas higiênicas de conduta. Sua lógica é a mesma que orientou toda a vida social da. China, no período em que foi desenvolvida: o calendário agrícola, as festas coletivas, os princípios de comportamento social, as regras de etiqueta no trato com as autoridades, a religião, a música, a arquitetura... Os princípios teóricos a partir dos quais as doenças são entendidas, classificadas e tratadas são os mesmos que servem para entender, classificar e lidar com as coisas do mundo', a natureza, o espaço e o tempo. (A este respeito ver: Granet, 1968).

Pretender que a eficácia de um saber que, segundo Cai Jing Feng, "tem controlado as maiores epidemias de doenças infecciosas na história da China", deva-se a que a introdução de agulhas, em determinados pontos, tenha como conseqüência a liberação de mediadores bioquímicos que interferem no fenômeno da dor; e que o sucesso obtido pelos chineses com a acupuntura
(*) Em nossa opiniăo, tanto a simplicidade quanto o baixo custo atribuldos à acupuntura são muito mais reflexo da falta de conhecimento mais profundo do método, do que características reais da medicina tradicional chinesa, 
(*) O Yiang e Yin constituem um par de opostos complementares a que săo relacionadas as dualidades/díades da natureza: dia $x$ noite, claro $x$ escuro, calor $x$ frio, masculino $x$ feminino, resisténcia $x$ complacéncia, movimento $x$ repouso, ... Granet reconhece o Yin e o Yang como 'símbolos' de grande poder sugestivo, uma vez que, constituindo uma dupla, evocam, agrupando em pares, todos os outros sfmbolos'.

"E o Tao que faz surgir ora - obscuro (Yin) ora o luminoso (Yang)" diz o I Ching (TA TAUN, Cap. 5), Granet comen. th as diferentes possibilidades de traduçāo da expressảo "Uma vez Yin, Uma vez Yang, e o Tao" - "primeiro o Yin, depois o Yang...", ou "aqui o Yin, lá o Yang...", ou "um tempo Yin, um tempo Yang...", ou, ainda, "um lado Yin, um lado Yang..." (p. 104) - segundo o autor, como o Yin e o Yang, o Tao é uma categoria concreta", uma totalidade "alternante e clclica", um "poder regulador" que "nảo cria os seres, os faz ser como săo", e que "regula o ritmo das coisas". (p. 269). De acordo com a tradiçâo: "O Tao possui todas as coisas em plena abun. dância, este é o seu campo de ação. Renova todas as coisas, todos os dias; este é o seu glorioso poder." (I Ching, TA TAUN cap. 5). durante dois mil e quinhentos anos de desenvolvimento seja fruto apenas da acumulação de observaçōes empíricas, é fechar os olhos ao saber tradicional, é descaracterizá-lo, é optar por uma 'cegueira etnocêntrica'.

Embora os autores orientais considerem que a política de integração entre os dois sistemas, praticada na China desde 1949 , tenha permitido um grande desenvolvimento não só na atenção à saúdé do povo chinês, como da própria medicina tradicional - e 0 surgimento das técnicas de anestesia cirúrgica com acupuntura nos anos cinqüenta testemunha que têm razẫo -, a recíproca pode nāo ser verdadeira.

Nos últimos quarenta anos, o oriente desenvolveu novas técnicas terapêuticas, associando o saber milenar com o saber e a técnica ocidentais. Ao ocidente, que não domina o saber tradicional, - ao contrário, o nega - restou a pobre e limitada perspectiva de procurar esclarecer, com seus próprios recursos teóricos, os mecanismos de ação e os efeitos de uma técnica oriental isolada em uma situação específica, a dor. No ocidente, a procura da cientificidade da acupuntura, ao contrário de esclarecer (ou legitimar) o saber que lhe dá sentido, tem sido a busca da confirmação da hegemonia da ciência médica, a possibilidade de fazê-la capaz de explicar os efeitos até então enigmáticos das agulhas.

Se o crescimento da aceitação da acupuntura no ocidente pode ser o reflexo da crise da medicina científica, e se a crise da medicina ocidental se identifica com a crise de seu paradigma positivista; então os estudos 'científicos' da acupuntura pouco poderāo contribuir para a superaçāo dessa crise, enquanto insistirem em negar a possibilidade de uma medicina, de uma ciência de dois mil e quinhentos anos, que tem a sua lógica própria, diferente daquela da ciência ocidental.

É possivel (quiçá provável) que a maior colaboração que o oriente possa trazer à medicina ocidental não esteja na sua técnica, mas no seu saber, nos conceitos a respeito da natureza e da causalidade das doenças, na sua visão holística do ser humano, na valorização da tendência à autocura inerente ao organismo, no significado do "equilíbrio" que se busca com a terapia.

No entanto, para se ter acesso ao saber tradicional, será preciso compreender os seus princípios, poder perceber claramente o Yin e o Yang no mundo, e a possibilidade do Tao $(*)$; será preciso reconhecer na natureza, no céu e na terra, as seis energias e os cinco elementos, aprender a distinguir, no exame do paciente, onde está o frio e onde está o calor, onde estão as insuficiências e onde estão os excessos.

Cadernos de Saúde Pública, RJ, 6 (2) : 117-128, abr/jun, 1990 
Para se ter acesso ao saber tradicional, será preciso admitir a possibilidade de que estas categorias possam se organizar em um sistema coerente cuja lógica, que orientou tanto a ordenação biológica quanto a ordenação social da China, durante a maior parte dos últimos vinte e cinco séculos, deve ser apreendida não só pelo estudo da medicina, como pela compreensão da religião, da filosofia, dos costumes, enfim, da história e da cultura da civilização chinesa.

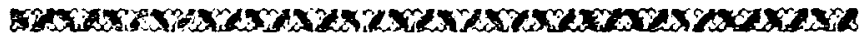

The recognition of acupuncture's effectiveness doesn't depend on the empirical demonstration of its results. There are methodological and conceptual barriers to the establishment of its therapeutical value, on a scientific basis.

On the other hand, the increase of demand and supply of alternative therapies implies in a certain

legitimation, which depends rather on the recognition of the utility of these practices, than on the demonstration of its scientific status.

The crisis of the "scientific medicine" and its mechanistic paradigm may be one of the factors influencing the acceptation of acupuncture in the West. If this is true, scientific studies on acupuncture won't be very useful unless they admit the possibility of a medicine which has its own logic, different from the western science.

Perhaps the greatest contribution brought by the East to the western medicine is its knowledge, and not its technique. But it's only through the understanding of the chinese culture and civilization, the acceptance that Yin and Yang form a coherent system, that the tradicional knowledge can be really seized.

\section{REFERÊNCIAS BIBLIOGRÁFICAS}

BLAND, J. - Uma ciência exata, A Saúde do Mundo, OMS, Dez/1979.

FENG, Cai Jing - Integration of traditional chinese medicine with western medicine: right or wrong?, Soc. Sci. Med. 27 (5), 1988.

CAPRA, F. - O ponto de mutação, Ed. Cultrix, São Paulo, 1986.

CINTRACT, M. - Curso rápido de acupuntura, Ed. Andrei, São Paulo, 1982.

Cademos de Saúde Pública, RJ, 6 (2) : 117-128, abr/jun, 1990 
GRANET, M. - La pensée chinoise, Ed. Albin Michel, Paris, 1968.

MING, Huan Xing - Novas esperanças, A Salude do Mundo, OMS, Dez/1979.

KAO, F. - O assunto da moda, A Saúde do Mundo, OMS, Dez/1979.

LEWITH, G. T. - Can we assess the effects of acupuncture? British Medical Journal, Vol. 288, 19, may, 1984.

PATEL, M. S. - Problems in the evaluation of alternative medicine, Soc. Sci. Med., (25) 6, 1987.

QUEIROZ, M. S. - O paradigma mecanicista da medicina ocidental moderna: uma perspectiva antropológica, Rev. Saúde Pública São Paulo 20(4), 1986.

WILHELM, R. - I Ching: o livro das mutaçöes, Ed. Pensamento, Săo Paulo, s/d.

YEKUTIEL, P. - Lecciones que se derivan de las grandes campañas de erradicación, Foro Mundial de la Salud, 2(4), 1981. 